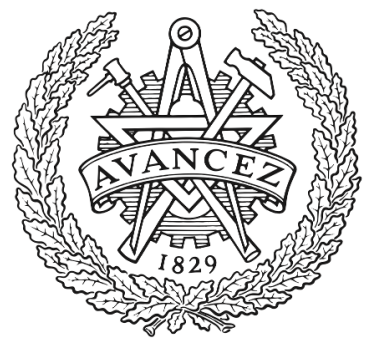

CHALMERS

UNIVERSITY OF TECHNOLOGY

\title{
Quantum description and emergence of nonlinearities in strongly coupled single-emitter nanoantenna systems
}

Downloaded from: https://research.chalmers.se, 2023-04-26 06:29 UTC

Citation for the original published paper (version of record):

Rousseaux, B., Baranov, D., Käll, M. et al (2018). Quantum description and emergence of nonlinearities in strongly coupled single-emitter nanoantenna systems. PHYSICAL REVIEW B, 98(4).

http://dx.doi.org/10.1103/PhysRevB.98.045435

N.B. When citing this work, cite the original published paper. 


\title{
Quantum description and emergence of nonlinearities in strongly coupled single-emitter nanoantenna systems
}

\author{
Benjamin Rousseaux, ${ }^{1,2,{ }^{*}}$ Denis G. Baranov, ${ }^{1}$ Mikael Käll, ${ }^{1}$ Timur Shegai, ${ }^{1}$ and Göran Johansson ${ }^{2}$ \\ ${ }^{1}$ Department of Physics, Chalmers University of Technology, 41296 Göteborg, Sweden \\ ${ }^{2}$ Department of Microtechnology and Nanoscience-MC2, Chalmers University of Technology, 41296 Göteborg, Sweden
}

(Received 5 April 2018; revised manuscript received 4 July 2018; published 31 July 2018)

\begin{abstract}
Realizing strong coupling between a single quantum emitter (QE) and an optical cavity is of crucial importance in the context of various quantum optical applications. Although Rabi splitting of single quantum emitters coupled to high- $Q$ classical cavities has been reported in numerous configurations, attaining single emitter Rabi splitting with a plasmonic nanostructure remains a challenge. In particular, strong coupling at the single QE regime would open the path for the realization of single-photon nonlinearities. In this paper, we derive a plasmon quantization procedure for systems consisting of a single QE located in the gap of a nanoantenna. This procedure leads to the description of the quantum dynamics by a master equation for the state of the QE and the quantized plasmonic modes, which is crucial to demonstrate the emergence of single-photon nonlinearities. We investigate numerically the optical response and the resulting Rabi splitting in metallic nanoantennas and find the optimal geometries for the emergence of the strong-coupling regime with single QEs. Finally, we demonstrate the saturation of hybridized modes for a chosen configuration. Our results will be useful for implementation of realistic quantum plasmonic nanosystems involving single QEs at room temperature.
\end{abstract}

DOI: 10.1103/PhysRevB.98.045435

\section{INTRODUCTION}

Interaction between the electromagnetic modes of a cavity and a two-level quantum emitter (QE) in the most simple picture described by the Jaynes-Cummings (JC) Hamiltonian is responsible for a rich variety of peculiar quantum optical effects [1-3]. The Rabi frequency $\Omega$, which in this framework determines the strength of interaction between light and matter, is the crucial parameter determining the behavior of a strongly coupled system. The weak light-matter interaction, realized when the Rabi frequency is small compared to rates of dissipative processes, manifests itself in the Markovian dynamics of the system characterized by the irreversible spontaneous decay of the QE $[4,5]$. It can be further accelerated by increasing the local density of optical states (LDOS), e.g., using a cavity [6-10].

Strong coupling, on the other hand, is a special regime of light-matter interaction, emerging when the Rabi frequency exceeds the rates of incoherent processes [11-14]. In this regime of light-matter interaction the photonic and matter components of the system can no longer be treated as separate entities as they form the polaritonic states (sometimes referred to as the dressed states) with their eigenenergies being separated by the Rabi splitting of $\Omega[12,15-17]$. Such an evolution of the system spectrum modifies its response and dramatically affects transport [18,19] and chemical [20-23] properties. Strong light-matter coupling is particularly interesting in the single emitter limit when unique features of the Jaynes-Cummings ladder enable ultrafast and single-photon optical nonlinearities $[24,25]$.

*benjamin.rousseaux@chalmers.se
Although strong coupling between high- $Q$ dielectric cavities and single emitters, such as atoms, quantum dots, and superconducting qubits has been the subject of intense research [26-29], much less progress has been made in the realization of strong coupling between single emitters and plasmonic nanostructures. The ability to strongly couple a single QE to a nanoscale plasmonic antenna would be extra beneficial for quantum information processing applications [30-33]. Although observations of Rabi splitting with ensembles of quantum emitters coupled to plasmonic structures have been widely reported [13,34-39], only a few recent reports claimed observation of Rabi splitting with a single QE [40-44]. Achieving prominent and robust splittings in plasmonic structures is hindered mostly by low- $Q$ factors of such nanocavities-a problem that has been suggested to deal with via structuring the environment of the emitter $[45,46]$.

Reaching the regime of strong coupling with a single QE is challenging. Indeed, several previous theoretical studies have described a related but less demanding regime of interaction, i.e., the emergence of Fano resonance in single QE-plasmon systems [47-49]. Such phenomena can also lead to single-photon nonlinearities on the nanoscale with the saturation of the QE when the system is driven strongly enough. However, Fano interference requires both high quantum yield of the nanoantenna and low internal loss of the $\mathrm{QE}$, thus low-temperature setups. Strongly coupled single QE-plasmon systems would enable the observation of singlephoton nonlinearities at room temperature as the QE drastically affects the optical response of the plasmonic structure. Studies reporting strong coupling between a single $\mathrm{QE}$ and the plasmonic cavities have revealed characteristic values for both the Purcell factors and the QE dipole moments involved in this regime $[40,50-56]$. 
In this paper, we systematically address the problem of strong coupling with a single $\mathrm{QE}$ within a quantum description of plasmonic resonances. In Sec. II, we derive a quantization procedure based on Refs. [57-59] and extend it to an arbitrary shaped nanoantenna by linking the Jaynes-Cummings coupling $g$ with numerical solutions of the local density of states. The effective non-Hermitian Hamiltonian is then shown to be equivalent to a quantum master equation description of the QE-plasmon system where the plasmonic mode is described as a mixture of bright and dark modes. In Sec. III we establish the analytical condition for strong coupling between a single QE and a plasmonic nanocavity and analyze various plasmonic nanostructures enabling Rabi splitting. We study numerically the optical response and the resulting Rabi splitting in metallic nanostructures, such as bow-tie nanoantennas, nanosphere dimers, and nanospheres on a surface and find the optimal geometries for the emergence of the strong-coupling regime with single QEs corresponding to several existing materials. We also discuss the impact of multiple modes of a plasmonic resonator on the strong-coupling regime and the trade-off between radiative and nonradiative processes. Finally, we demonstrate the emergence of a single-photon nonlinearity by numerically solving the master equation for an optimal configuration, and calculate the second-order correlation function $g^{(2)}(\tau)$ for this configuration. In the last section, we summarize our results and prospects in a conclusion.

\section{QUANTUM DESCRIPTION AND STRONG-COUPLING REGIME}

\section{A. LDOS and Purcell factor}

In this section, we develop the theoretical framework for the description of a single QE coupled to a nanoscale structure. This framework is based on the description of spontaneous emission in a structured environment, which is originally pictured by the Purcell effect, as the QE decays faster into an environment with a higher number of surrounding modes. We derive the quantization procedure leading to a set of specific cavity modes associated with localized surface-plasmon resonances, enabling the construction of the master equation for the dynamics and derive the threshold condition for the LDOS and the QE dipole moment upon which strong coupling occurs between the QE and the cavity modes (see Fig. 1).

We start with the formulation of the LDOS, which is a spacedependent quantity describing how fast quantum emitters can decay in localized field modes. It is determined with the Green's tensor of the electric-field contribution, corresponding to the solution of the Maxwell-Helmholtz equation,

$$
\left(\nabla \times \nabla \times-\frac{\omega^{2}}{c^{2}} \epsilon(\mathbf{r}, \omega)\right) \overline{\bar{G}}_{\omega}\left(\mathbf{r}, \mathbf{r}^{\prime}\right)=\overline{\bar{I}} \delta\left(\mathbf{r}-\mathbf{r}^{\prime}\right),
$$

where $\epsilon(\mathbf{r}, \omega)$ is the dielectric function and $\overline{\bar{I}}$ is the $3 \times 3$ identity matrix. For an emitter whose transition dipole moment is along the unit vector $\mathbf{n}$, the LDOS is as follows:

$$
\rho_{\mathbf{n}}\left(\mathbf{r}_{E}, \omega\right)=\frac{6 \omega}{\pi c^{2}} \mathbf{n} \cdot \operatorname{Im}\left\{\overline{\bar{G}}_{\omega}\left(\mathbf{r}_{E}, \mathbf{r}_{E}\right)\right\} \mathbf{n},
$$

where $\mathbf{r}_{E}$ is the position of the emitter and $\omega$ is the angular frequency. We also consider the quantity defined through the

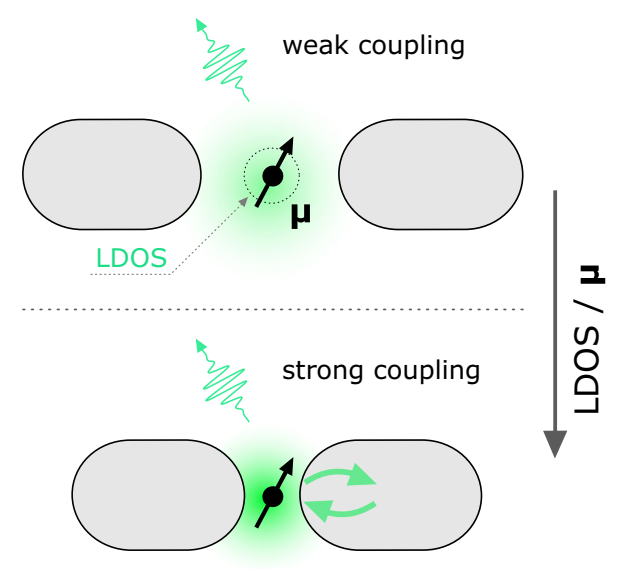

FIG. 1. Schematic of the structure under study: a two-level system modeling a quantum emitter positioned in the hot spot of a nanoantenna. Upon an increase in the LDOS or the QE transition dipole moment, the system exhibits transition from the weak- to strong-coupling regime when Rabi oscillations take over spontaneous emission.

relation,

$$
F_{P}(\omega)=\frac{\Gamma}{\gamma_{0}}=\frac{\rho_{\mathbf{n}}\left(\mathbf{r}_{E}, \omega\right)}{\rho_{\mathbf{n}}^{0}\left(\mathbf{r}_{E}, \omega\right)}
$$

which is the Purcell factor at frequency $\omega$, and $\rho_{\mathbf{n}}^{0}\left(\mathbf{r}_{E}, \omega\right)$ is the LDOS in free space. It can also be written in terms of the modified spontaneous emission rate $\Gamma$ of the QE, divided by its free space spontaneous emission rate $\gamma_{0}$. We note that the Purcell factor is a property of the nanoantenna alone, hence it is independent of the QE dipole moment. It is seen from (2) that the knowledge of the Green's tensor $\overline{\bar{G}}_{\omega}\left(\mathbf{r}_{E}, \mathbf{r}_{E}\right)$ provides the LDOS and the Purcell factor. The Green's dyadic can be obtained in two manners: Using analytical derivations, and using numerics. In the case of spherical and spheroidal geometries (e.g., for spherical or prolate spheroidal nanoparticles), the Green's tensor can be obtained analytically $[60,61]$. Nevertheless, for more complicated geometries, one has to rely on numerical calculations [62]. The tools for getting the Green's function of arbitrary geometries are provided by finite-difference time-domain (FDTD) software.

\section{B. Quantum description of a single emitter coupled to plasmonic modes \\ 1. General Green's tensor approach}

To understand the different coupling regimes, we use a quantum description and derive an effective Hamiltonian [57-59] whose structure is completely analogous to cavity QED Hamiltonians. The derivation is based on a first-principles method corresponding to the coupling of a single two-level system with a reservoir of harmonic oscillators: the Fano diagonalization $[63,64]$. In this description, the electric field is toggled by creation and annihilation operators $\widehat{\mathbf{f}}_{\omega}^{\dagger}(\mathbf{r}), \widehat{\mathbf{f}}_{\omega}(\mathbf{r})$ obeying the commutation relations,

$$
\left[\widehat{\mathbf{f}}_{\omega}(\mathbf{r}), \widehat{\mathbf{f}}_{\omega^{\prime}}^{\dagger}\left(\mathbf{r}^{\prime}\right)\right]=\delta\left(\mathbf{r}-\mathbf{r}^{\prime}\right) \delta\left(\omega-\omega^{\prime}\right)
$$


In other words, the environment of the quantum emitter corresponding to the nanoparticles and the surrounding free space is diagonalized and described by these global operators. In addition, the geometry of the structure considered is reflected in the use of the Green's tensor $\overline{\bar{G}}_{\omega}\left(\mathbf{r}, \mathbf{r}^{\prime}\right)$ in the expression of the electric field,

$$
\begin{aligned}
\widehat{\mathbf{E}}(\mathbf{r}) & =\int_{0}^{+\infty} d \omega\left[\widehat{\mathbf{E}}_{\omega}^{(+)}(\mathbf{r})+\widehat{\mathbf{E}}_{\omega}^{(-)}(\mathbf{r})\right] \\
\widehat{\mathbf{E}}_{\omega}^{(+)}(\mathbf{r}) & =i \sqrt{\frac{\hbar}{\pi \epsilon_{0}}} \frac{\omega^{2}}{c^{2}} \int d^{3} r^{\prime} \sqrt{\epsilon_{\omega}^{\prime \prime}\left(\mathbf{r}^{\prime}\right)} \overline{\bar{G}}_{\omega}\left(\mathbf{r}, \mathbf{r}^{\prime}\right) \cdot \widehat{\mathbf{f}}_{\omega}\left(\mathbf{r}^{\prime}\right) \\
\widehat{\mathbf{E}}_{\omega}^{(-)}(\mathbf{r}) & =\left[\widehat{\mathbf{E}}_{\omega}^{(+)}(\mathbf{r})\right]^{\dagger}
\end{aligned}
$$

where we introduced the imaginary part of the dielectric function $\epsilon_{\omega}^{\prime \prime}(\mathbf{r})=\operatorname{Im}\{\epsilon(\mathbf{r}, \omega)\}$, which contains the information about the optical properties of the geometry. The calculation of the Green's tensor along with the boundary conditions projects the general structure of the field into a subspace corresponding to the specific choice of the geometry. In this consideration the basis of the creation-annihilation operators $\widehat{\mathbf{f}}_{\omega}^{\dagger}(\mathbf{r}), \widehat{\mathbf{f}}_{\omega}(\mathbf{r})$ is also projected onto a subspace of electromagnetic modes, and the field Hamiltonian reduces to the basis,

$$
\begin{aligned}
\widehat{H}_{\text {field }} & =\int_{0}^{+\infty} d \omega \hbar \omega \int d^{3} r \widehat{\mathbf{f}}_{\omega}^{\dagger}(\mathbf{r}) \widehat{\mathbf{f}}_{\omega}(\mathbf{r}) \\
& \longrightarrow \sum_{\eta} \int_{0}^{+\infty} d \omega \hbar \omega \widehat{a}_{\eta}^{\dagger}(\omega) \widehat{a}_{\eta}(\omega),
\end{aligned}
$$

where here the creation-annihilation operators $\widehat{a}_{\eta}^{\dagger}(\omega), \widehat{a}_{\eta}(\omega)$ toggle excitations of localized surface-plasmon cavity modes labeled by the general index $\boldsymbol{\eta}$ at frequency $\omega$. For the new operators to obey the commutation relation $\left[\widehat{a}_{\eta}(\omega), \widehat{a}_{\eta^{\prime}}^{\dagger}\left(\omega^{\prime}\right)\right]=$ $\delta_{\eta \eta^{\prime}} \delta\left(\omega-\omega^{\prime}\right)$, it is required that all cavity modes $\eta$ are orthogonal to each other. As an example, in the case of an emitter close to a nanosphere, this general index reduces to a harmonic index $n=1,2, \ldots$, whose values are associated with specific mode geometries: $n=1$ is the dipolar mode, $n=2$ is the quadrupolar mode, etc. [57,58]. The coupling of the plasmonic field and the emitter is introduced with a dipole coupling term $-\widehat{\boldsymbol{\mu}} \cdot \widehat{\mathbf{E}}\left(\mathbf{r}_{E}\right)$ where the $\mathrm{QE}$ is a pointlike two-level quantum system with a finite dipole moment $\mu$, and the total rotating-wave approximation (RWA) Hamiltonian reads

$$
\begin{aligned}
\widehat{H}= & \hbar \omega_{0} \widehat{\sigma}_{+} \widehat{\sigma}_{-}+\sum_{\eta} \int_{0}^{+\infty} d \omega \hbar \omega \widehat{a}_{\eta}^{\dagger}(\omega) \widehat{a}_{\eta}(\omega) \\
& +i \hbar \sum_{\eta} \int_{0}^{+\infty} d \omega\left[\kappa_{\eta}(\omega) \widehat{a}_{\eta}^{\dagger}(\omega) \widehat{\sigma}_{-}-\text {H.c. }\right] .
\end{aligned}
$$

In the above expression, $\omega_{0}$ denotes the transition frequency of the two-level emitter, $\widehat{\sigma}_{-}=|g\rangle\langle e|, \widehat{\sigma}_{+}=\widehat{\sigma}_{-}^{\dagger}$ are the lowering and raising operators of the transition, and $\kappa_{\eta}(\omega)$ is the emittermode coupling, associated with a specific mode $\eta$. The latter contains the Green's tensor through the expression,

$$
\left|\kappa_{\eta}(\omega)\right|^{2}=\frac{1}{\hbar \pi \epsilon_{0}} \frac{\omega^{2}}{c^{2}} \boldsymbol{\mu} \cdot \operatorname{Im}\left\{\overline{\bar{G}}_{\eta, \omega}\left(\mathbf{r}_{E}, \mathbf{r}_{E}\right)\right\} \boldsymbol{\mu},
$$

with $\overline{\bar{G}}_{\boldsymbol{\eta}, \omega}\left(\mathbf{r}_{E}, \mathbf{r}_{E}\right)$ being the Green's tensor corresponding to mode $\boldsymbol{\eta}$. We see that the square modulus of the emitter-field coupling is proportional to the LDOS (2) for a given mode. The knowledge of the latter is then essential for the understanding of the quantum description.

\section{Single-mode effective model}

In cavity QED, the description of the cavity field is often, to a good approximation, taken to be a single mode. In quantum plasmonics, however, this is often not the case, and one has to include many plasmonic modes in the model as is the case for spherical nanoparticles. The single-mode approach can nevertheless be a useful tool for understanding the different coupling regimes.

Let us consider a single-mode $\boldsymbol{\eta}$ interacting with a $\mathrm{QE}$. The corresponding emitter-mode coupling $\kappa(\omega)$ has a Lorentzian shape [57,58],

$$
\kappa(\omega)=\sqrt{\frac{\gamma_{\text {cav }}}{2 \pi}} \frac{g}{\omega-\omega_{c}+i \frac{\gamma_{\text {cav }}}{2}},
$$

where $\omega_{c}$ is the cavity-mode frequency, $\gamma_{\text {cav }}$ is the cavity decay rate being equal to the full width of its scattering spectrum, and $g$ is the usual Jaynes-Cummings interaction constant,

$$
g=\sqrt{\frac{\pi \omega_{c} \gamma_{\mathrm{cav}} \rho_{\mathbf{n}}\left(\mathbf{r}_{E}, \omega_{c}\right)}{12 \hbar \epsilon_{0}}} \mu .
$$

Solving the time-dependent Schrödinger equation with Hamiltonian (7) and using the Lorentzian profile (9), it can be shown that the full Hamiltonian reduces to the effective Hamiltonian,

$$
\widehat{H}_{\text {eff }}=\left(\begin{array}{cc}
\omega_{0} & g \\
g & \omega_{c}-i \frac{\gamma_{\text {cav }}}{2}
\end{array}\right),
$$

which is expressed in the single excitation basis $\{|e, 0\rangle,|g, 1\rangle\}$ where only one photon is exchanged via the $|e\rangle \leftrightarrow|g\rangle$ transition. This effective Hamiltonian is non-Hermitian due to the loss terms on the diagonal as this arises when coupling a discrete state coupled to a continuum of modes. It is also equivalent to take only the Hermitian part of this Hamiltonian and write a Lindblad master equation for the time evolution of the state $[65,66]$,

$\dot{\widehat{\varrho}}=-i\left[\widehat{H}_{\mathrm{JC}}, \widehat{\varrho}(t)\right]+\gamma_{\mathrm{cav}}\left(\widehat{a} \widehat{\varrho}(t) \widehat{a}^{\dagger}-\frac{1}{2} \widehat{a}^{\dagger} \widehat{a} \widehat{\varrho}(t)-\frac{1}{2} \widehat{\varrho}(t) \widehat{a}^{\dagger} \widehat{a}\right)$,

where $\widehat{\varrho}(t)$ is the density operator for the state of the QEplasmon system, $\widehat{a}$ is the annihilation operator of the plasmon mode, and $\widehat{H}_{\mathrm{JC}}=\omega_{0} \widehat{\sigma}_{+} \widehat{\sigma}_{-}+\omega_{c} \widehat{a}^{\dagger} \widehat{a}+g\left(\widehat{a}^{\dagger} \widehat{\sigma}_{-}+\widehat{a} \widehat{\sigma}_{+}\right)$is the Jaynes-Cummings Hamiltonian. We must underline that Eq. (12) has no damping term corresponding to the two-level $\mathrm{QE}$ and that all the dissipation is channeled through the Lindblad operator $\widehat{L}=\sqrt{\gamma_{\text {cav }}} \widehat{a}$ corresponding to the electromagnetic environment of the QE. In particular, it is shown that in the Purcell regime $\left(\gamma_{\text {cav }} \gg g\right)$, the master equation reduces to [67]

$$
\begin{aligned}
\widehat{\varrho}_{0}= & -i\left[\widehat{H}_{0}, \widehat{\varrho}_{0}(t)\right]+\frac{4 g^{2}}{\gamma_{\text {cav }}}\left(\widehat{\sigma}_{-} \widehat{\varrho}_{0}(t) \widehat{\sigma}_{+}-\frac{1}{2} \widehat{\sigma}_{+} \widehat{\sigma}_{-} \widehat{\varrho}_{0}(t)\right. \\
& \left.-\frac{1}{2} \widehat{\varrho}_{0}(t) \widehat{\sigma}_{+} \widehat{\sigma}_{-}\right),
\end{aligned}
$$


where $\widehat{\varrho}_{0}=\operatorname{Tr}_{\text {cav }}\{\widehat{\varrho}\}$ is the reduced density operator associated with the QE and $\widehat{H}_{0}=\omega_{0} \widehat{\sigma}_{+} \widehat{\sigma}_{-}$is the free QE Hamiltonian. Moreover it can be easily seen that replacing the LDOS in (10) by the free space LDOS $\rho_{\mathbf{n}}^{0}\left(\mathbf{r}_{E}, \omega_{0}\right)=\omega_{0}^{2} / \pi^{2} c^{3}$ and taking $\omega_{c}=\omega_{0}$, the decay rate $4 g^{2} / \gamma_{\text {cav }}$ reduces to the free space decay rate,

$$
\gamma_{0}=\frac{\mu^{2} \omega_{0}^{3}}{3 \hbar \pi \epsilon_{0} c^{3}}
$$

\section{STRONG-COUPLING CONDITION, COMPARATIVE STUDY, AND SINGLE-PHOTON NONLINEARITY}

\section{A. Analytical condition}

The dynamics of a single QE coupled to a plasmonic mode is parametrized by three quantities: the JC interaction constant $g$, the QE free space decay rate $\gamma_{0}$, and the cavity mode decay rate $\gamma_{\text {cav }}$. In the weak-coupling regime, when $g \ll \gamma_{\text {cav }}$, the QE experiences spontaneous emission: The probability to find the QE in the excited-state $P_{e}(t)$ decays exponentially in time with the rate $\gamma_{0} F_{P}\left(\omega_{0}\right)$, where $F_{P}$ is the well-known Purcell factor, which reflects acceleration of the spontaneous emission due to enhanced local density of states [6-8]. In the singlemode approximation and assuming that the $\mathrm{QE}$ is resonant with the cavity-mode $\omega_{c}=\omega_{0}$, the Purcell factor can be expressed through the interaction constant via [2]

$$
F_{P}=\frac{4 g^{2}}{\gamma_{\text {cav }} \gamma_{0}}
$$

As the interaction constant increases, $g \sim \gamma_{\text {cav }}$ or even $g>$ $\gamma_{\text {cav }}$, single photons start to oscillate between the emitter and the mode, giving rise to non-Markovian dynamics of the emitter population $P_{e}(t)$, which is a signature of the strong-coupling regime. Mathematically, strong coupling occurs whenever separation between the two eigenvalues of Hamiltonian (11) exceeds $\left(\gamma_{\text {cav }}+\gamma_{0}\right) / 2$. In general, they should even exceed $\left(\gamma_{\text {cav }}+\gamma\right) / 2$, where $\gamma=\gamma_{0}+\gamma_{\text {inh }}$ is the total decay rate of the transition, accounting for both free space and inhomogeneous decay. For simplicity, we will consider $\gamma_{\text {inh }}=0$ in this paper. Comparing the two eigenvalues of (11) (assuming that the QE is resonant with the nanoantenna mode, $\omega_{0}=\omega_{c}$, and $\gamma_{\text {cav }} \gg$ $\gamma_{0}$ ), we find that the onset of strong coupling corresponds to the interaction constant $g=\gamma_{\text {cav }} /(2 \sqrt{2})$. Correspondingly, estimating the Purcell factor $F_{P}$ at the onset of strong coupling, we obtain a convenient threshold condition for strong coupling in terms of the Purcell factor,

$$
F_{P}\left(\omega_{0}\right) \geqslant \frac{\gamma_{\mathrm{cav}}}{2 \gamma_{0}} .
$$

Typical values of the plasmon lifetime and the QE lifetime lie on the scale of $10 \mathrm{fs}$ and $1 \mathrm{~ns}$, respectively. The latter equation thus suggests that $F_{P}$ should be at least on the order of $10^{5}$ in order to reach strong coupling with a single $\mathrm{QE}$ coupled to a realistic plasmon resonator.

In order to reach strong coupling, the interaction constant $g$ should be increased. This can be performed in two different manners: either by increasing the transition dipole moment $\mu$ of the $\mathrm{QE}$ or by increasing the Purcell factor $F_{P}$. The set of available QEs is often limited to a few options. Much greater flexibility is, however, offered by designing the optical cavity and maximizing the Purcell factor.

Expression (16) allows one to estimate the threshold magnitude of the dipole moment $\mu_{\text {s.c. }}$ of a single QE which is required to reach strong coupling with a given nanoantenna with some value of $F_{P}$. To do so, we note that the free space spontaneous emission rate $\gamma_{0}$ in Eq. (16) depends on $\mu$; expressing $\mu$ from this formula yields us the threshold dipole moment magnitude,

$$
\mu_{\text {s.c. }}=\sqrt{\frac{3 \hbar \pi \epsilon_{0} c^{3} \gamma_{\mathrm{cav}}}{2 \omega_{0}^{3} F_{P}}}
$$

This expression can be used to find the optimal geometry for reaching the strong-coupling regime with a single emitter: One needs to calculate the Purcell factor and the cavity decay rate and check if the resulting values are enough to guarantee strong coupling with a given emitter. However, we underline that we limit our approach with a point-dipole approximation for the QE in order to provide a general description for the single emitter regime. In general the size of the QE leads to the breakdown of this approximation when it is comparable to the dimensions of the antenna tips, and recent studies have presented a time-dependent density functional theory approach to calculate the coupling $g$ beyond the point-dipole approximation [68].

\section{B. Comparative study of the nanoantenna geometry}

Now we employ the condition (17) to study the feasibility of reaching the strong-coupling regime in various plasmonic nanoantennas with single QEs. We will consider three nanoantenna types: (i) a dimer of two nanospheres, (ii) a nanoparticle on a surface (NPoS), and (iii) a bow-tie nanoantenna. We also consider two materials from which antennas are made: gold $(\mathrm{Au})$ and silver $(\mathrm{Ag})$ for the bow tie (resonances in the other two Ag antennas are strongly shifted to the UV region which makes them less interesting). Nanometer gaps in these antennas ensure deeply subwavelength mode volumes and high Purcell factors lowering the required transition dipole moment. To study the coupling in these nanoantennas, we calculated numerically the LDOS spectra using the Lumerical FDTD solver. The mesh step around the gap where the point source was located was set to $0.1 \mathrm{~nm}$. Convergence of simulations was ensured by performing simulations for gradually reducing the mesh step and monitoring the difference of the result with the result of the previous simulation.

The maximum of each spectrum is identified as the plasmonic mode, and a corresponding QE resonant with that mode is assumed in further calculations. The LDOS spectra for each geometry considered here are presented in Fig. 2 for 3-nm gaps in all cases. It is seen that the LDOS of bow-tie nanoantennas around the bright mode is a single Lorentzian, but this is not the case for the NPoS and sphere dimers due to the presence of other strongly nonradiative modes. The calculation shown in Fig. 2 is then performed for different gap sizes using the same geometries.

The calculated spectra are then fitted with an effective Lorentzian function (9) parametrized by the width $\gamma_{\text {cav }}$, the Jaynes-Cummings coupling $g$, and the resonance frequency $\omega_{c}$. Replacing the full LDOS by a single Lorentzian fit works 

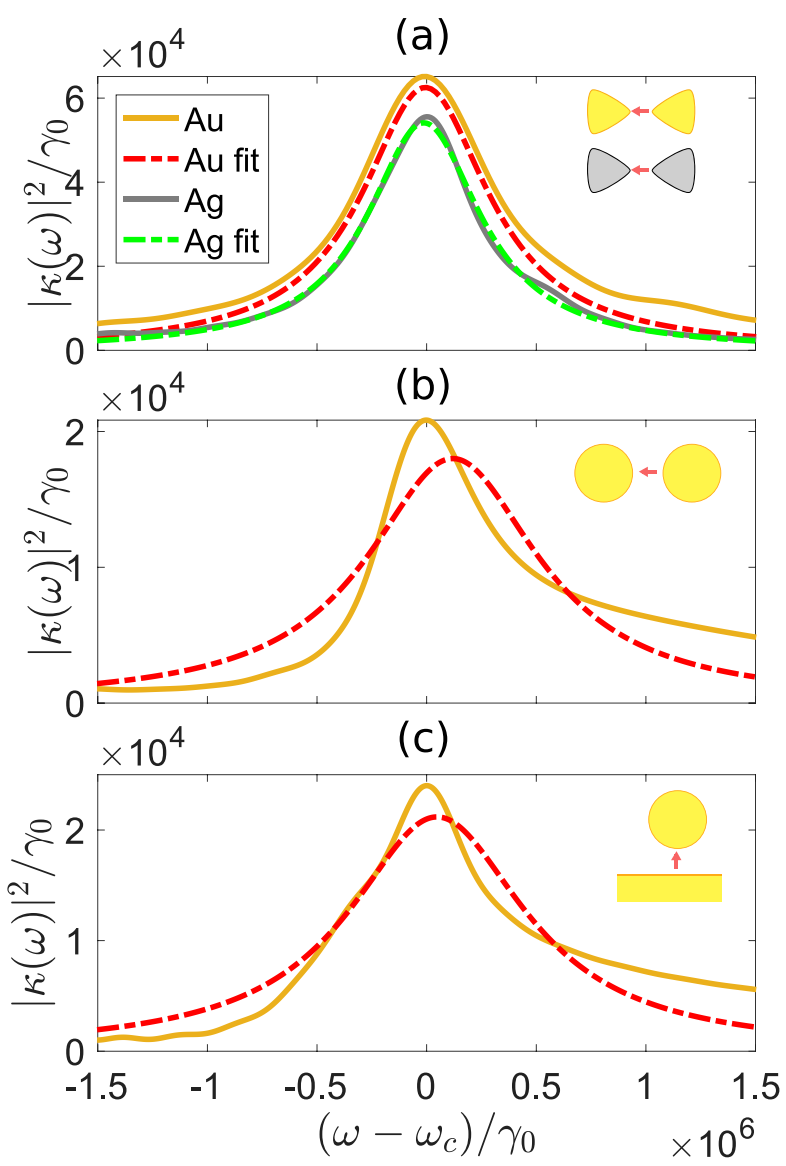

(d)

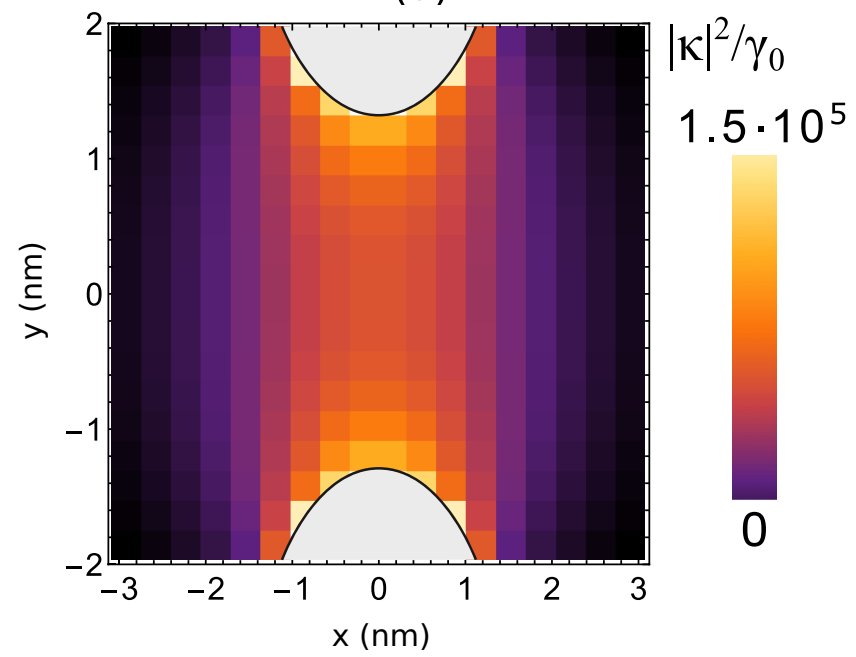

FIG. 2. (a)-(c) FDTD calculation of the QE-plasmon coupling $|\kappa(\omega)|^{2}$ proportional to the LDOS at the center of the gap (solid blue) and Lorentzian fit of the considered modes (dashed-dotted red). The calculation is performed for four different geometries. (a) Au and Ag bow-tie nanoantennas. (b) Au nanosphere dimer. (c) Au NPoS. The permittivity of $\mathrm{Ag}$ and $\mathrm{Au}$ was adopted from Ref. [69]. For bow-tie nanoantennas, triangles with $90-\mathrm{nm}$ side lengths, 50-nm bases, and 10 -nm thicknesses were used. For the NPoS, nanospheres with 50-nm diameters were used. For the dimer, 30-nm nanospheres were used. The gaps in all cases were set to $3 \mathrm{~nm}$. (d) Spatial map of the total Purcell factor for an Ag bow-tie nanoantenna with a 3-nm gap at resonance $(\sim 750 \mathrm{~nm})$ for an electric dipole oriented along the gap.

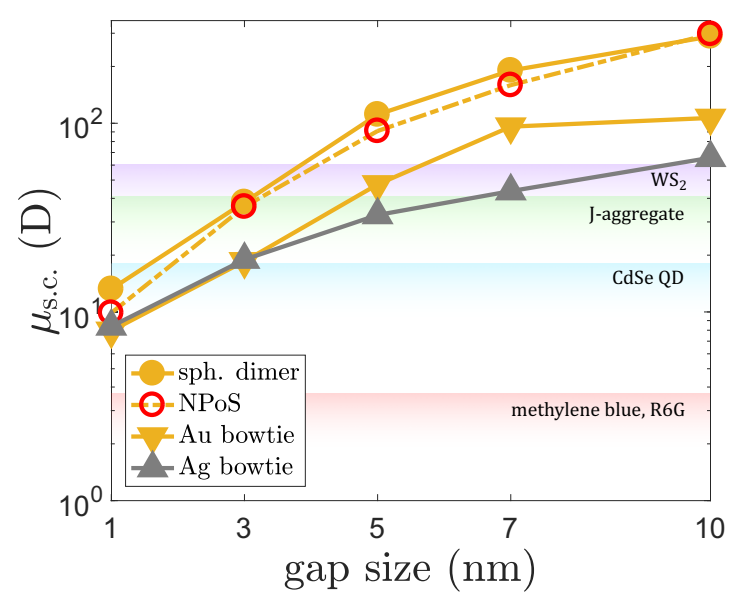

FIG. 3. Minimal dipole moment (17) for reaching the strongcoupling regime in debyes (D). The calculations are performed for $\mathrm{Au}$ and $\mathrm{Ag}$ bow ties, the Au NPoS, and the Au nanosphere dimer.

well for the bow-tie nanoantennas, Figs. 2(a) and 2(b). In the case of the NPoS and nanosphere dimers, however, the total LDOS does not feature a Lorentzian behavior. Nevertheless, for simplicity we fit the LDOS with a Lorentzian peak using the least-squares method as we are interested in the global coupling strength between the nanoantenna and the QE.

The LDOS and hence the coupling strength vary with the position at which the QE is located with respect to the nanoantenna. This is illustrated in Fig. 2(d) for the Ag bow-tie nanoantenna with a 3-nm gap for a QE's dipole moment oriented along the gap. The Purcell factor reaches the maximal values close to the antenna edges and quickly decays away from the edges. This plot also emphasizes that positioning of a $\mathrm{QE}$ exactly in the center of a gap is neither necessary nor optimal for ensuring the highest LDOS and interaction constant. Having performed the fitting procedure for each LDOS spectrum, we now calculate the threshold dipole moment $\mu_{\text {s.c. }}$ of a resonant $\mathrm{QE}$ to reach the strong-coupling regime for each nanoantenna. The results are presented in Fig. 3. Expectedly, the threshold dipole moment quickly decreases with shrinking gap for all antennas. Among all studied geometries, the bow-tie antennas exhibit the lowest threshold dipole moment, which is due to the lowest resonance width $\gamma_{\text {cav }}$ and a tightly localized electric field at the tips of the bow-tie nanoantenna. In the same plot we show horizontal bars corresponding to a few QEs often employed in the studies of strong light-matter interaction. The key observation is that QEs, such as excitons in monolayer $\mathrm{WS}_{2}$ and J-aggregates, may allow us to reach strong coupling in the single emitter limit with bow-tie nanoantennas with gaps as large as $5 \mathrm{~nm}$. As the gap shrinks down to $1 \mathrm{~nm}$, all four systems exhibit comparable responses with simple organic molecules, such as methylene blue, being on the edge of strong coupling. Note that for gaps smaller than $5 \mathrm{~nm}$, the nonlocal response of the metal may become pronounced, whereas our calculations of the coupling strength are based on the local model. This may have an unfavorable effect on the coupling strength as recent theoretical efforts suggest [70].

We further inspect the radiative properties of the four considered geometries. For the Rabi splitting to be clearly observable in the emission spectrum, the decay of a QE must 
TABLE I. Quantum yield $\eta$ of an emitter placed in the center of the gap tuned to the antenna resonance for the four studied nanoantennas.

\begin{tabular}{lcccc}
\hline \hline Nanoantenna & NPoS & Nanosphere dimer & Au bow tie & Ag bow tie \\
\hline$\eta$ & 0.0015 & 0.0026 & 0.2796 & 0.4513 \\
\hline \hline
\end{tabular}

be mostly radiative. This can be quantified by the quantum yield of the QE,

$$
\eta=\frac{\Gamma_{\mathrm{rad}}}{\Gamma_{\mathrm{tot}}}
$$

where $\Gamma_{\text {rad }}$ is the radiative decay rate of the QE in the vicinity of the nanoantenna. The quantum yield of an emitter located at the gap center at the resonance wavelength for each nanoantenana is presented in Table I. The bow-tie geometry exhibits the best radiative properties among the studied antennas, orders of magnitude higher than the NPoS and the nanosphere dimer do. The spatial dependence of the quantum yield for the $\mathrm{Ag}$ bow tie shown in Fig. 4 suggests that the quantum yield is sensitive to the emitter position, but even very close to the metal edges the quantum yield reduces only down to 0.1 , which is still 500 to 1000 times higher than the NPoS and the nanosphere dimer value. Together with the result shown in Fig. 2(d) it highlights the fact the precise positioning of a $\mathrm{QE}$ at a very specific site around the nanoantenna is not that crucial for reaching strong coupling as one might anticipate.

\section{Single QE nonlinearity in a bow-tie nanoantenna}

Finally, to support our claims, we demonstrate the feasibility of strong coupling with a bow-tie nanoantenna and a single emitter via simulating the temporal dynamics of the emitter population. We choose a $\mathrm{WS}_{2}$ exciton with the transition dipole moment of $50 \mathrm{D}[71,72]$ as a single $\mathrm{QE}$ and tune the Ag bow-tie resonance to the $\mathrm{WS}_{2}$ monolayer exciton transition wavelength of $\approx 610 \mathrm{~nm}$. Figure 5 shows the temporal dynamics of the $\mathrm{QE}$ population associated with the $\mathrm{Ag}$ bow-tie nanoantenna studied in Figs. 2 and 3, and clearly shows that at least one Rabi cycle occurs until the emitter irretrievably decays to the ground state.

Whereas the calculation shown in Fig. 5 is performed considering a single excitation in the $\mathrm{QE}$ as an initial condition, that is, $|\psi(0)\rangle=|e\rangle \otimes|0\rangle$, the dynamics can also be determined

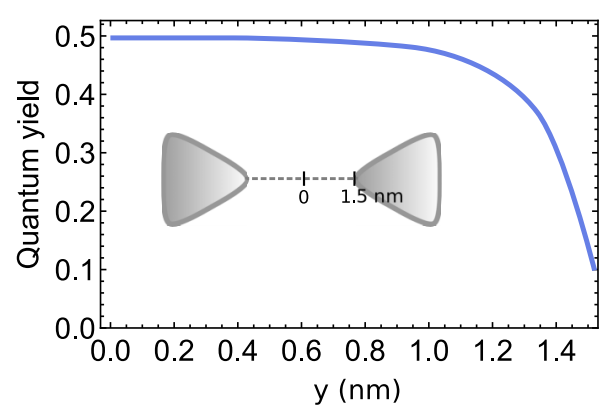

FIG. 4. Spatial dependence of the quantum yield of a resonant emitter interacting with a 3-nm gap Ag bow-tie nanoantenna. The emitter wavelength is $610 \mathrm{~nm}$, and the dipole moment is oriented in the $y$ direction. The origin is chosen to be 0 , and $y=1.5 \mathrm{~nm}$ corresponds to the tip of one nanoprism.

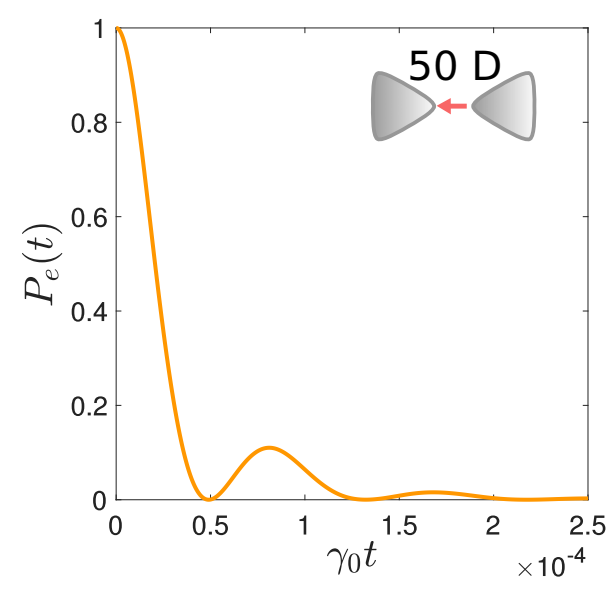

FIG. 5. Calculated temporal dynamics of the population of a QE with a 50-D transition dipole moment corresponding to the $\mathrm{WS}_{2}$ exciton. The antenna geometry is tuned for its resonance to match the $\mathrm{WS}_{2}$ exciton wavelength of $610 \mathrm{~nm}$.

using the steady-state solution of the master equation (12). To do so we replace $\widehat{H}_{\mathrm{JC}}$ with a new Hamiltonian $\widehat{H}^{\prime}=$ $\widehat{H}_{\mathrm{JC}}^{\prime}+\widehat{H}_{\text {pump }}$ in a rotating frame oscillating with the pump frequency $\omega_{P}$ such that we have the effective RWA terms,

$$
\begin{aligned}
\widehat{H}_{\mathrm{JC}}^{\prime} & =\Delta_{0} \widehat{\sigma}_{+} \widehat{\sigma}_{-}+\Delta_{c} \widehat{a}^{\dagger} \widehat{a}+g\left(\widehat{a}^{\dagger} \widehat{\sigma}_{-}+\widehat{a} \widehat{\sigma}_{+}\right), \\
\widehat{H}_{\text {pump }} & =\frac{\mathcal{E}}{2}\left(\widehat{a}+\widehat{a}^{\dagger}\right),
\end{aligned}
$$

where $\Delta_{0, c}=\omega_{0, c}-\omega_{P}$ and $\mathcal{E}=-\boldsymbol{\mu}_{\text {n.a. }} \cdot \mathbf{E}_{0} / \hbar$ is the Rabi frequency associated with the pump laser field $\mathbf{E}_{P}(t)=$ $\mathbf{E}_{0} \cos \omega_{P} t$ and the effective transition dipole moment of the nanoantenna $\mu_{\text {n.a. }}$. The steady state of the density operator is determined taking the time derivative in (12) being equal to zero and finding the nontrivial solutions of the equation,

$$
\mathcal{L} \varrho_{\text {s.s. }}=0,
$$

$\mathcal{L}=i\left[\widehat{H}^{\prime}, \bullet\right]+\gamma_{\text {cav }}\left(\widehat{a} \bullet \widehat{a}^{\dagger}-\frac{1}{2} \widehat{a}^{\dagger} \widehat{a} \bullet-\frac{1}{2} \bullet \widehat{a}^{\dagger} \widehat{a}\right)$ being the superoperator acting on the density operator. The spectra are then computed using the average formula $\langle\widehat{A}\rangle=\operatorname{Tr}\left\{\varrho_{\text {s.s. }} \widehat{A}\right\}$.

We evaluate the steady-state mode populations in Fig. 6, for three different quantities: the intensity of the cavity field $\left\langle\widehat{a}^{\dagger} \widehat{a}\right\rangle$, the QE contribution $\left\langle\widehat{\sigma}_{+} \widehat{\sigma}_{-}\right\rangle$, and their corresponding polaritonic mixture $\left\langle\widehat{\Lambda}_{ \pm}^{\dagger} \widehat{\Lambda}_{ \pm}\right\rangle$where the upper $(+)$and lower $(-)$ polariton operators are given by

$$
\widehat{\Lambda}_{ \pm}=\frac{1}{\sqrt{2}}\left(\widehat{a} \pm \widehat{\sigma}_{-}\right) \text {. }
$$

The mode populations are calculated for three pump Rabi frequency values of $\mathcal{E}=(0.1,1,2) \gamma_{\mathrm{cav}}$, and the saturation phenomenon is shown to happen for the two higher values. In particular, the plasmonic cavity Lorentzian line shape is recovered for $\mathcal{E}=2 \gamma_{\text {cav }}$, whereas the $\mathrm{QE}$ contribution is reduced and recombines as a single peak for the same value. We also see the same behavior in the polariton populations $\left\langle\widehat{\Lambda}_{ \pm}^{\dagger} \widehat{\Lambda}_{ \pm}\right\rangle$, which we display as an interesting way to separate the lower and upper components in the dynamics.

Finally, we compute the photon statistics for the same configuration using the $g^{(2)}(\tau)$ function, corresponding to a 

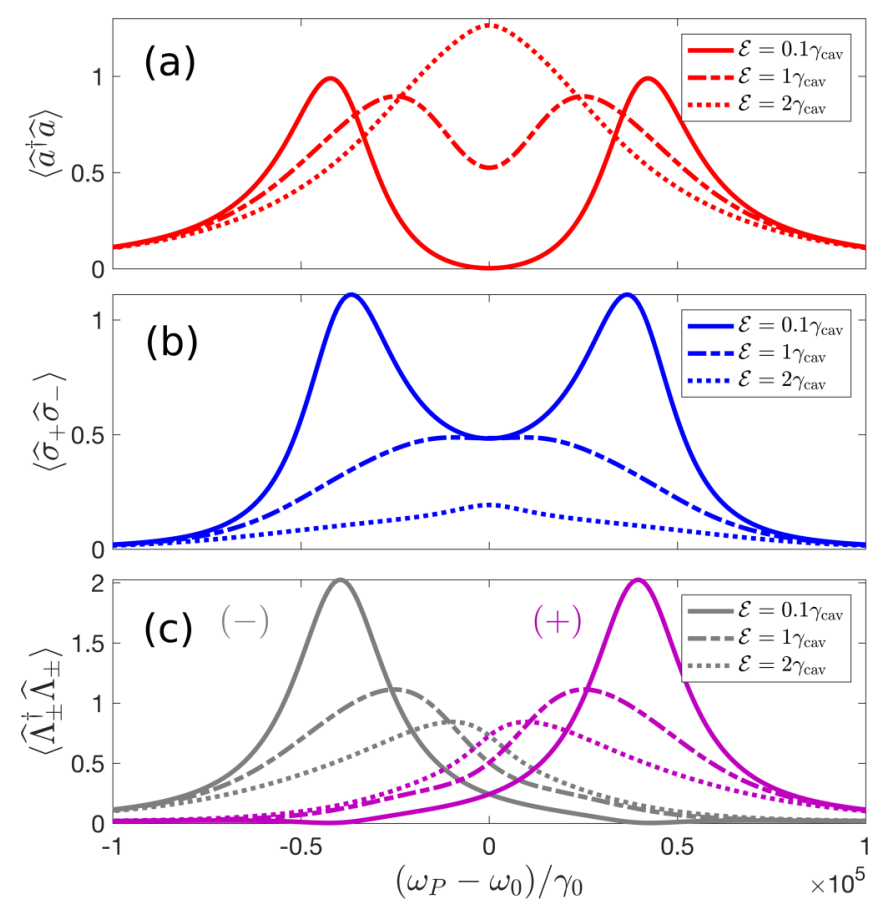

FIG. 6. Steady-state mode populations of a Ag bow tie and single QE system precedingly studied in Fig. 5 versus pump frequency $\omega_{P}$ for different values of the pump Rabi frequency: $\mathcal{E}=0.1 \gamma_{\text {cav }}$ (solid curves), $\gamma_{\text {cav }}$ (dashed-dotted curves), and $2 \gamma_{\text {cav }}$ (dotted curves). (a) Plasmonic resonance population $\left\langle\widehat{a}^{\dagger} \widehat{a}\right\rangle$. (b) QE excitation population $\left\langle\widehat{\sigma}_{+} \widehat{\sigma}_{-}\right\rangle$. (c) Polariton populations $\left\langle\widehat{\Lambda}_{ \pm}^{\dagger} \widehat{\Lambda}_{ \pm}\right\rangle$from the lower (gray) and upper (purple) polaritons. $y$ axes are in units of $1 / \mathcal{E}^{2}$.

Hanbury-Brown-Twiss experimental setup [see Fig. 7(a)],

$$
g^{(2)}(\tau)=\frac{\left\langle\widehat{a}_{\text {out }}^{\dagger}(t) \widehat{a}_{\text {out }}^{\dagger}(t+\tau) \widehat{a}_{\text {out }}(t+\tau) \widehat{a}_{\text {out }}(t)\right\rangle}{\left\langle\widehat{a}_{\text {out }}^{\dagger}(t) \widehat{a}_{\text {out }}(t)\right\rangle^{2}},
$$

where we introduced the output photon annihilation operator $\widehat{a}_{\text {out }}$, whose expression is given by the input-output relation [2],

$$
\widehat{a}_{\text {out }}=\widehat{a}_{\text {in }}+\sqrt{\gamma_{\text {cav }, \mathrm{r}} \widehat{a}},
$$

where $\gamma_{\mathrm{cav}, \mathrm{r}}$ is the radiative decay of the nanoantenna. This function is defined as the probability of detecting a photon at time $t+\tau$ whereas another photon was detected at time $t$, normalized by the probabilities of detecting a single photon at the same times. Nonclassical features of light are revealed when $g^{(2)}(\tau)<1$, and, in particular, antibunching is observed when $g^{(2)}(0)<1$. In that case, photons tend to be emitted one by one. Note that the $\widehat{a}_{\text {in }}$ term is disregarded as we consider only the scattered part and that linking expression (23) with the

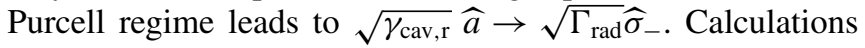
of the $g^{(2)}(\tau)$ function are shown in Fig. 7(c) for low pump $\left(\mathcal{E}=0.1 \gamma_{\text {cav }}\right)$. The red lines show the calculation for the $\mathrm{Ag}$ bow tie coupled to a 50-D QE, and oscillations due to the QE-plasmon coupling $g$ arise in the statistics, suppressing the antibunching when the system is pumped at $\omega_{P}=\omega_{c}-g$. This is due to the small splitting observed between the upper and the lower polariton states, and for larger splitting, it is seen that the oscillations are faster and averaged into an overall smooth behavior. However, if the pump is slightly redshifted to the lower polariton $\left|{ }_{1}\right\rangle$ [red dashed-dotted line in Fig. 7(c)], it (a)

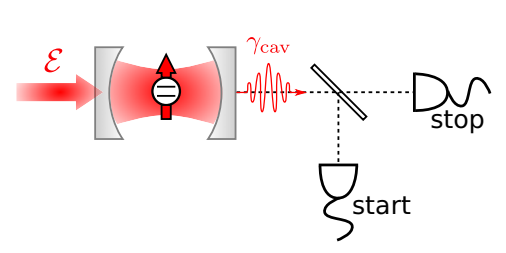

(c)

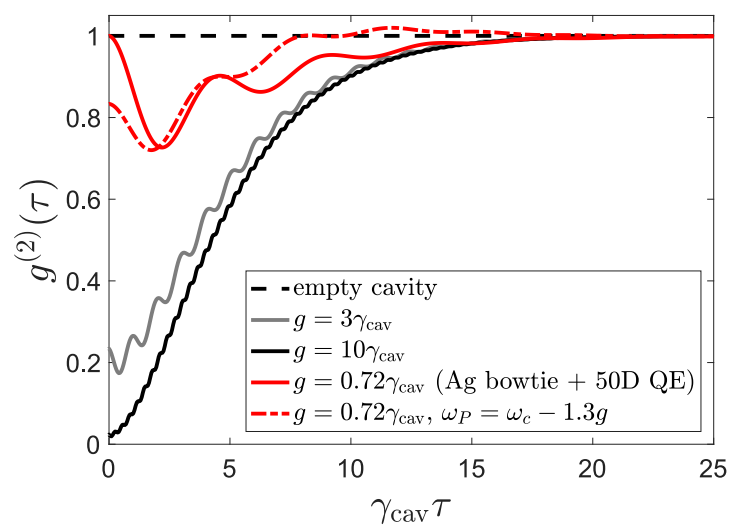

FIG. 7. (a) Hanbury-Brown-Twiss setup: A cavity containing a $\mathrm{QE}$ is coherently pumped with the laser Rabi frequency $\mathcal{E}=0.1 \gamma_{\text {cav }}$ at frequency $\omega_{P}$, and the output field is split into two beams hitting avalanche photodiodes. (b) Linkage pattern of the single-photon Jaynes-Cummings ladder with the groundstate $|g, 0\rangle$ and the polariton states $\left| \pm_{1}\right\rangle=\widehat{\Lambda}_{ \pm}^{\dagger}|g, 0\rangle$. The lower polariton is pumped at frequency $\omega_{P}=\omega_{c}-g$. (c) The $g^{(2)}(\tau)$ function for different $g / \gamma_{\text {cav }}$ ratios. The red lines correspond to the Ag bow tie with $50 \mathrm{D}$.

is seen that $g^{(2)}(0)<1$ and therefore a small antibunching is observed. We emphasize that the calculation of the photon statistics is performed assuming that it is possible to correlate the output signals. Nevertheless, the time scale observed here is on the order of $\gamma_{\text {cav }} \tau \sim 5$, which is about $30 \mathrm{fs}$. Even though it is challenging to access $g^{(2)}(\tau)$ at this fast time scale, it is however interesting to underline the nonclassical nature of the light emitted by a QE coupled to a nanoantenna. If this is performed properly, the antibunching can be observed on the nanosecond to picosecond time scales.

\section{CONCLUSION}

To conclude, we have systematically analyzed various plasmonic nanoantennas in the context of the Rabi splitting with single quantum emitters using a fully quantized electromagnetic-field response. We established the general condition that can be used to estimate the feasibility of the strong-coupling regime and applied it to study the required dipole moment of a single emitter in order to achieve the strong-coupling regime. We have found that silver and gold bow-tie nanoantennas present the most favorable structures for attaining strong coupling with single emitters. The conditions seem to be especially viable for the case of single excitons of two-dimensional semiconductors, such as $\mathrm{WS}_{2}$. Finally, we demonstrated numerically the emergence of single-photon nonlinearities with the saturation and photon statistics of a 
strongly coupled system made of a QE placed in the gap of a silver bow-tie nanoantenna using a master equation formalism. Future research should be aimed at exploring even more beneficial nanoantenna geometries and addressing single excitons in two-dimensional materials. This opens the perspective for realizing broadband photon-photon interactions on the nanoscale.

\section{ACKNOWLEDGMENTS}

The authors acknowledge financial support from the Knut and Alice Wallenberg Foundation. T.S. and G.J. acknowledge financial support from the Swedish Research Council (VR Grant No. 2016-06059).
[1] E. T. Jaynes and F. W. Cummings, Proc. IEEE 51, 89 (1963).

[2] M. O. Scully and M. S. Zubairy, Quantum Optics (Cambridge University Press, Cambridge, UK, 1997), p. 656.

[3] S. M. Dutra, Cavity Quantum Electrodynamics: The Strange Theory of Light in a Box (Wiley, Hoboken, NJ, 2005).

[4] V. Weisskopf and E. Wigner, Z. Phys. 63, 54 (1930).

[5] L. Allen and J. H. Eberly, Optical Resonance and Two-Level Atoms (Wiley, New York, 1975).

[6] E. M. Purcell, Phys. Rev. 69, 681 (1946).

[7] M. Pelton, Nat. Photonics 9, 427 (2015).

[8] D. G. Baranov, R. S. Savelev, S. V. Li, A. E. Krasnok, and A. Alu, Laser Photon. Rev. 11, 1600268 (2017).

[9] K. J. Russel, T. L. Liu, S. Cui, and E. L. Hu, Nat. Photonics 6, 2091 (2012).

[10] N. P. de Leon, B. J. Shields, C. L. Yu, D. E. Englund, A. V. Akimov, M. D. Lukin, and H. Park, Phys. Rev. Lett. 108, 226803 (2012).

[11] H. J. Kimble and T. W. Lynn, Coherence and Quantum Optics, (Kluwer Academic/Plenum, Dordrecht, 2003).

[12] G. Khitrova, H. M. Gibbs, M. Kira, S. W. Koch, and A. Scherer, Nat. Phys. 2, 81 (2006).

[13] P. Törmä and W. L. Barnes, Rep. Prog. Phys. 78, 013901 (2015).

[14] D. G. Baranov, M. Wersall, J. Cuadra, T. J. Antosiewicz, and T. Shegai, ACS Photonics 5, 24 (2018).

[15] J. M. Fink, M. Goppl, M. Baur, R. Bianchetti, P. J. Leek, A. Blais, and A. Wallraff, Nature (London) 454, 315 (2008).

[16] L. S. Bishop, J. M. Chow, J. Koch, A. A. Houck, M. H. Devoret, E. Thuneberg, S. M. Girvin, and R. J. Schoelkopf, Nat. Phys. 5, 105 (2009).

[17] J. Kasprzak, S. Reitzenstein, E. A. Muljarov, C. Kistner, C. Schneider, M. Strauss, S. Hofling, A. Forchel, and W. Langbein, Nature Mater. 9, 304 (2010).

[18] J. Schachenmayer, C. Genes, E. Tignone, and G. Pupillo, Phys. Rev. Lett. 114, 196403 (2015).

[19] J. Feist and F. J. Garcia-Vidal, Phys. Rev. Lett. 114, 196402 (2015).

[20] J. A. Hutchison, T. Schwartz, C. Genet, E. Devaux, and T. W. Ebbesen, Angew. Chem., Int. Ed. Engl. 51, 1592 (2012).

[21] A. Thomas, J. George, A. Shalabney, M. Dryzhakov, S. J. Varma, J. Moran, T. Chervy, X. Zhong, E. Devaux, C. Genet, J. A. Hutchison, and T. W. Ebbesen, Angew. Chem., Int. Ed. Engl. 55, 11462 (2016).

[22] F. Herrera and F. C. Spano, Phys. Rev. Lett. 116, 238301 (2016).

[23] J. Galego, F. J. Garcia-Vidal, and J. Feist, Nat. Commun. 7, 13841 (2016).

[24] K. M. Birnbaum, A. Boca, R. Miller, A. D. Boozer, T. E. Northup, and H. J. Kimble, Nature (London) 436, 87 (2005).

[25] D. Englund, A. Faraon, I. Fushman, N. Stoltz, P. Petroff, and J. Vuckovic, Nature (London) 450, 857 (2007).
[26] J. P. Reithmaier, G. Sek, A. Löffler, C. Hofmann, S. Kuhn, S. Reitzenstein, L. V. Keldysh, V. D. Kulakovskii, T. L. Reinecke, and A. Forchel, Nature (London) 432, 197 (2004).

[27] T. Yoshie, A. Scherer, J. Hendrickson, G. Khitrova, H. M. Gibbs, G. Rupper, C. Ell, O. B. Shchekin, and D. G. Deppe, Nature (London) 432, 200 (2004).

[28] A. Boca, R. Miller, K. M. Birnbaum, A. D. Boozer, J. McKeever, and H. J. Kimble, Phys. Rev. Lett. 93, 233603 (2004).

[29] E. Peter, P. Senellart, D. Martrou, A. Lemaître, J. Hours, J. M. Gérard, and J. Bloch, Phys. Rev. Lett. 95, 067401 (2005).

[30] M. S. Tame, K. R. McEnery, S. K. Ozdemir, J. Lee, S. A. Maier, and M. S. Kim, Nat. Phys. 9, 329 (2013).

[31] D. Dzsotjan, A. S. Sørensen, and M. Fleischhauer, Phys. Rev. B 82, 075427 (2010).

[32] T. Volz, A. Reinhard, M. Winger, A. Badolato, K. J. Hennessy, E. L. Hu, and A. Imamoğlu, Nat. Photonics 6, 605 (2012).

[33] H. Rangani Jahromi, Opt. Commun. 411, 119 (2018).

[34] N. T. Fofang, N. K. Grady, Z. Fan, A. O. Govorov, and N. J. Halas, Nano Lett. 11, 1556 (2011).

[35] A. E. Schlather, N. Large, A. S. Urban, P. Nordlander, and N. J. Halas, Nano Lett. 13, 3281 (2013).

[36] S. R. K. Rodriguez, J. Feist, M. A. Verschuuren, F. J. Garcia Vidal, and J. Gómez Rivas, Phys. Rev. Lett. 111, 166802 (2013).

[37] A. Cacciola, O. Di Stefano, R. Stassi, R. Saija, and S. Savasta, ACS Nano 8, 11483 (2014).

[38] G. Zengin, M. Wersäll, S. Nilsson, T. J. Antosiewicz, M. Käll, and T. Shegai, Phys. Rev. Lett. 114, 157401 (2015).

[39] G. Zengin, G. Johansson, P. Johansson, T. J. Antosiewicz, M. Käll, and T. Shegai, Sci. Rep. 3, 3074 (2013).

[40] T. Hartsfield, W.-S. Chang, S.-C. Yang, T. Ma, J. Shi, L. Sun, G. Shvets, S. Link, and X. Li, Proc. Natl. Acad. Sci. USA 112, 12288 (2015).

[41] K. Santhosh, O. Bitton, L. Chuntonov, and G. Haran, Nat. Commun. 7, ncomms11823 (2016).

[42] R. Chikkaraddy, B. de Nijs, F. Benz, S. J. Barrow, O. A. Scherman, E. Rosta, A. Demetriadou, P. Fox, O. Hess, and J. J. Baumberg, Nature (London) 535, 127 (2016).

[43] R. Liu, Z.-K. Zhou, Y.-C. Yu, T. Zhang, H. Wang, G. Liu, Y. Wei, H. Chen, and X.-H. Wang, Phys. Rev. Lett. 118, 237401 (2017).

[44] H. Groß, J. M. Hamm, T. Tufarelli, O. Hess, and B. Hecht, Sci. Adv. 4, eaar4906 (2018).

[45] P. Peng, Y.-C. Liu, D. Xu, Q.-T. Cao, G. Lu, Q. Gong, and Y.-F. Xiao, Phys. Rev. Lett. 119, 233901 (2017).

[46] B. Gurlek, V. Sandoghdar, and D. Martín-Cano, ACS Photonics 5, 456 (2017).

[47] X. Wu, S. K. Gray, and M. Pelton, Opt. Express 18, 23633 (2010).

[48] R. A. Shah, N. F. Scherer, M. Pelton, and S. K. Gray, Phys. Rev. B 88, 075411 (2013). 
[49] X.-W. Chen, V. Sandoghdar, and M. Agio, Phys. Rev. Lett. 110, 153605 (2013).

[50] A. Trügler and U. Hohenester, Phys. Rev. B 77, 115403 (2008).

[51] S. Savasta, R. Saija, A. Ridolfo, O. D. Stefano, P. Denti, and F. Borghese, ACS Nano 4, 6369 (2010).

[52] A. Manjavacas, F. J. G. de Abajo, and P. Nordlander, Nano Lett. 11, 2318 (2011).

[53] T. Hümmer, F. J. García-Vidal, L. Martín-Moreno, and D. Zueco, Phys. Rev. B 87, 115419 (2013).

[54] K. Słowik, R. Filter, J. Straubel, F. Lederer, and C. Rockstuhl, Phys. Rev. B 88, 195414 (2013).

[55] S. J. P. Kress, F. V. Antolinez, P. Richner, S. V. Jayanti, D. K. Kim, A. Riedinger, M. P. C. Fischer, S. Meyer, K. M. McPeak, D. Poulikakos, and D. J. Norris, Nano Lett. 15, 6267 (2015).

[56] F. Marquier, C. Sauvan, and J. J. Greffet, ACS Photonics 4, 2091 (2017).

[57] B. Rousseaux, D. Dzsotjan, G. Colas des Francs, H. R. Jauslin, C. Couteau, and S. Guérin, Phys. Rev. B 93, 045422 (2016).

[58] D. Dzsotjan, B. Rousseaux, H. R. Jauslin, G. C. des Francs, C. Couteau, and S. Guérin, Phys. Rev. A 94, 023818 (2016).

[59] H. Varguet, B. Rousseaux, D. Dzsotjan, H.-R. Jauslin, S. Guérin, and G. C. des Francs, Opt. Lett. 41, 4480 (2016).

[60] L.-W. Li, P.-S. Kooi, M.-S. Leong, and T.-S. Yeo, IEEE Trans. Microwave Theory Tech. 42, 2302 (1994).
[61] L.-W. Li, X.-K. Kang, and M.-S. Leong, Spheroidal Wave Functions in Electromagnetic Theory (Wiley, Hoboken, NJ, 2002).

[62] C. Sauvan, J. P. Hugonin, I. S. Maksymov, and P. Lalanne, Phys. Rev. Lett. 110, 237401 (2013).

[63] S. S. L. Knoll and D.-G. Welsch, Coherence and Statistics of Photons and Atoms, (Wiley, Hoboken, NJ, 2001).

[64] T. G. Philbin, New J. Phys. 12, 123008 (2010).

[65] B. M. Garraway, Phys. Rev. A 55, 2290 (1997).

[66] B. J. Dalton, S. M. Barnett, and B. M. Garraway, Phys. Rev. A 64, 053813 (2001).

[67] R. Azouit, A. Sarlette, and P. Rouchon, 55th IEEE Conference on Decision and Control, Las Vegas, 2016 (IEEE, Piscataway, NJ, 2016).

[68] T. Neuman, R. Esteban, D. Casanova, F. J. García-Vidal, and J. Aizpurua, Nano Lett. 18, 2358 (2018).

[69] P. B. Johnson and R.-W. Christy, Phys. Rev. B 6, 4370 (1972).

[70] R. Jurga, S. D’Agostino, F. Della Sala, and C. Ciracì, J. Phys. Chem. C 121, 22361 (2017).

[71] E. J. Sie, J. W. McIver, Y.-H. Li, L. Fu, J. Kong, and N. Gedik, Nature Mater. 14, 290 (2015).

[72] J. Cuadra, D. G. Baranov, M. Wersäll, R. Verre, T. J. Antosiewicz, and T. Shegai, Nano Lett. 18, 1777 (2018). 